\title{
A Novel Image Restoration Method based on Iterative Algorithm
}

\author{
Saiyan $\mathrm{Wu}$, Hui Yang \\ Taiyuan Institute of Technology, Taiyuan, 030008, China
}

Received: December 1, 2020. Revised: May 8, 2021. Accepted: May 31, 2021. Published: June 7, 2021.

\begin{abstract}
In the paper, we proposed a new iterative algorithm and use a entirely new iterative factor. Firstly, we adopt the Exp function in the iterative factor, because we want each iterative result preserves the nonnegative constraint; Secondly, we make the iterative factor in a reciprocal form ,this way can produce two advantages, one is we can get a more stable and continuous results after each iteration; the other is we can achieve this algorithm in hardware more convenient. Thirdly, we add a low-pass filter and the edge of the scale in the iterative factor, this way we can get a better result, the image SNR is higher and the MSE is lower. Meanwhile for the image sequence, we adopt the two-step iterative algorithm. The result shows the algorithm own the faster convergence speed and the better convergence result. Different from the other algorithm for blind restoration, although we should select the parameter in the starting of the algorithm, the algorithm doesn't sensitive for the parameter. So the algorithm possesses very strong adaptability for the blind image deblurring. So a novel algorithm based on an iterative and nonnegative algorithm was proposed to perform blind deconvolution.
\end{abstract}

Keywords - iterative algorithm, blind image deblurring, image process, image sequence.

\section{INTRODUCTION}

$\mathrm{S}$ ince image degradation is caused by convolution [1-2], the purpose of image deblurring is to eliminate convolution factor [3]. Due to the ill posed problems of deconvolution and noise in the observed image, it is necessary to improve the resolution of the image, which makes a trade-off between resolution and noise suppression necessary.

At present, image restoration algorithms in spatial domain are still in-depth research [4]. Among these methods, regularization method is the most representative. Regularization method is proposed for the ill-posed problem of the deconvolution [5-6]. To solve the problem, the constraints are made on the result with a priori knowledge of the original image, and are used to lead the result continuously depending on the observed data. The method based on the above idea can be called regularization method [7]. The image restoration algorithm of frequency domain is another kind of method.
According to the flat region and edge region of the image, the different frequency characteristics of the image are mapped, and the observed image is transformed into the frequency domain through the transmission model. After data processing in frequency domain, the results are converted to spatial domain. Among the frequency domain method, the classic method is the Wiener Filter [8-9]. Wiener filtering and Incremental Wiener filtering have been widely used in the image restoration algorithm. In recent year, the method of intelligent computation is also applied to the image restoration [10]. The problem of image restoration can be seen as a combinatorial optimization problem. There are a variety of intelligent computing methods which can be used to solve combinatorial optimization problems. Refs [11] have summarized computational intelligence methods in image processing. Hopfield Neural Network [12-13] is a new method to solve the image restoration problem of the objective function minimization. Recently, the research on the stochastic process become a hotspot [14], and the Gaussian random field theory and Markov field theory are widely used to image restoration [15], which provides a Bayesian theory for the image restoration framework. The probability distribution of the image model provides maximum likelihood and maximum posterior standard to estimate clear image. Usually, image restoration problem is transformed into Bayesian estimation problem by probability model. The Richardson-Lucy algorithm [16], Maximum Likelihood Estimation algorithm [17], and multiplicative iterative approach [18] are all based on the probabilistic model. Usually, the algorithms of restoration for multiply image frames based on the fact that the target image of the image sequence will not change greatly in short-time. By applying the neighbouring blurred frames in the sequence of short-exposure, Yamaguchi $\mathrm{M}$ et al obtained a better quality image of the target image estimation[19]. In fact, a continuous multi-frame degraded image provides more complementary information to restrain the result of restored image and reduce the possibility of meanless solution. Compared to the restoration of a single image, the restoration of multi-frame image has great advantages on the effectiveness and stability [20]. However, during the recovery computation, multi-frame algorithms require the data of multi-frame images, which lead to huge computation and storage.

In this paper, the proposed method can reduce the 
computation and improve the efficiency of the restoration comparing with the restoration of the single-frame. Compared to the existing image restoration algorithms, the method in this paper mainly has the following advantages. The algorithm is positive stable after each iteration and does not need any additional constraints. The algorithm has low computational complexity and is easy to be implemented by hardware. Because the boundary filter and Gaussian filter are added to the iterative factor, better edge preservation and noise suppression effect can be obtained, and the image with high signal-to-noise ratio can be obtained.

\section{ITERATIVE ALGORITHM}

A new method is proposed to solve the problem of multi frame image restoration. The algorithm can solve the problem of the effective convergence stability, meanwhile its parameter selection is simple, and due to the non-iterative restoration algorithm, it has an advantage of negative constraints compared to other image restoration algorithms based on probability model.

Bregman iterative algorithm is an iterative regularization method, which is used to study the total variation image denoising. At the same time, it is applied to the compressed sensing reconstruction problem, and good results are obtained. Then, the method is applied to image denoising based on wavelet, nonlinear inverse scale space and medical MRI image. Recently, Bregman iterative algorithm has become one of the most effective methods for solving norm optimization problems and related optimization problems.

For the Gaussian noise,

$$
P(y \mid o, h)=\frac{1}{\sqrt{2 \pi} \sigma} \exp \left\{-\frac{\left(\mathrm{y}-\mathrm{o}^{*} \mathrm{~h}\right)^{2}}{2 \sigma^{2}}\right\}
$$

$\sigma$ is a covariance for the noise, $y(x)$ is the degraded image, $o(x)$ is the initial image, $h(x)$ is the PSF function.

We take the logarithm for the formula(1) and then multiply $\sigma^{2}$ at both sides of the formula.

$$
\begin{aligned}
\log [P(y \mid o, h)] & =\log \left(\frac{1}{\sqrt{2 \pi} \sigma}\right)+\log \left[\exp \left\{-\frac{\left(\mathrm{y}-\mathrm{o}^{*} \mathrm{~h}\right)^{2}}{2 \sigma^{2}}\right\}\right] \\
\sigma^{2} \log [\mathrm{P}(\mathrm{y} \mid \mathrm{o}, \mathrm{h})] & =\sigma^{2} \log \left(\frac{1}{\sqrt{2 \pi} \sigma}\right)-\frac{\left(\mathrm{y}-\mathrm{o}^{*} \mathrm{~h}\right)^{2}}{2} \\
\mathrm{~J}(\mathrm{o}, \mathrm{h}) & \equiv \sigma^{2} \log [\mathrm{P}(\mathrm{y} \mid \mathrm{o}, \mathrm{h})] \\
& =-\frac{\left(\mathrm{y}-\mathrm{o}^{*} \mathrm{~h}\right)^{2}}{2}+C
\end{aligned}
$$

$C$ equals to $\sigma^{2} \log \left(\frac{1}{\sqrt{2 \pi} \sigma}\right)$, which is constant and independent on $o(x)$ and $h(x)$.

The formula (3) can be obtained when $\lambda$ is a factor

$$
\begin{aligned}
& \frac{2}{1+e^{-\lambda_{1} h^{c *}[\mathrm{y}-\mathrm{o} * \mathrm{~h}]}}=1 \\
& \frac{2}{1+e^{-\lambda_{2} o^{c *}[\mathrm{y}-\mathrm{o} * \mathrm{~h}]}}=1
\end{aligned}
$$

When formula (3) is processed, formula (4) can be obtained

$$
\begin{aligned}
& \mathrm{o}_{k+1}=\mathrm{o}_{k} \frac{2}{1+\exp \left(-\lambda_{1} h_{k}{ }^{c} *\left[\mathrm{y}-\mathrm{o}_{k}{ }^{*} \mathrm{~h}_{k}\right]\right)} \\
& \mathrm{h}_{k+1}=h_{k} \frac{2}{1+\exp \left(-\lambda_{2} o_{k+1}{ }^{c} *\left[\mathrm{y}-\mathrm{o}_{k+1} * \mathrm{~h}_{k}\right]\right)}
\end{aligned}
$$

In the following modification, we need the iteration towards the effective convergence results. For the formula (4), we don't need the sufficient prior knowledge, choosing initial $o(x)$ and $h(x)$ for whole one mask, we can also get a convergence results. Meanwhile we should adjust the above formula (4) in order to maintain the edge details of the original image in each iteration, we add the factor vectors. In order to filter out noise in the end of each iteration, we add a Gaussian low-pass filter, then we obtain the final iterative equation.

$\mathrm{o}_{k+1}=\mathrm{o}_{k} \frac{2}{1+\exp \left(-\lambda_{1} h_{k}^{c} *\left[\mathrm{y}-\mathrm{o}_{k}{ }^{*} \mathrm{~h}_{k} *\left(1+\mu^{*} \text { edgevector }\right)\right] * \text { lowpass }\right)}$

$\mathrm{h}_{k+1}=h_{k} \frac{2}{1+\exp \left(-\lambda_{2} o_{k+1}{ }^{c} *\left[\mathrm{y}-\mathrm{O}_{k+1}{ }^{*} \mathrm{~h}_{k} *\left(1+\mu^{*} \text { edgevector }\right)\right] * \text { lowpass }\right)}$

Where $\mu$ is the edge coefficient, and values generally between $0.25-0.5$. When the image has rich edge details, the larger value is selected, otherwise the smaller value is selected. Edgevector is the sobel filter which is constant to the scale. In order to facilitate the calculation, the signals are stored directly in the form of frequency domain. lowpass is the Gaussian filter to filter high frequency noise components. In the experiment, the edgevector of $3 * 3$ Sober gradient operator and the lowpass of Gaussian low-pass filter are obtained. $\lambda$ is the convergence coefficient, when it takes a large value, the convergence speed will be very fast, but if the value is too large, it may not converge.

The algorithm process is described as follows.

STEP1. Choose appropriate parameter $\mu$ and $\lambda$.

STEP2. Choose the previous $N$ (generally $N$ between2-5) frames of the image sequence and the initial guesses of $\mathrm{O}_{k}(\mathrm{x})$ and $\mathrm{h}_{k}(\mathrm{x})$. In order to maintain the real-time, initial $o(x)$ and $h(x)$ are chosen for whole one mask, and then their volumes are normalized to unit one.

STEP3. Obtain $\mathrm{O}_{k+1}(\mathrm{x})$ and $\mathrm{h}_{k+1}(\mathrm{x})$ by formula (5) and (6). 
STEP4. If the specified iteration number $J$ is completed, stop the iteration, then compute the result of the original frame. Otherwise, return to STEP 3.

STEP5. Use the previous estimate result to provide the initial guesses of $\mathrm{O}_{k}(\mathrm{x})$ and $\mathrm{h}_{k}(\mathrm{x})$ for current frame.

STEP6. Obtain $\mathrm{o}_{k+1}(\mathrm{x})$ and $\mathrm{h}_{k+1}(\mathrm{x})$ by formula (5) and (6).

STEP7. If the specified iteration number $K$ is completed, stop the iteration, then compute the result of the prevent frame. Otherwise, return to STEP 6.

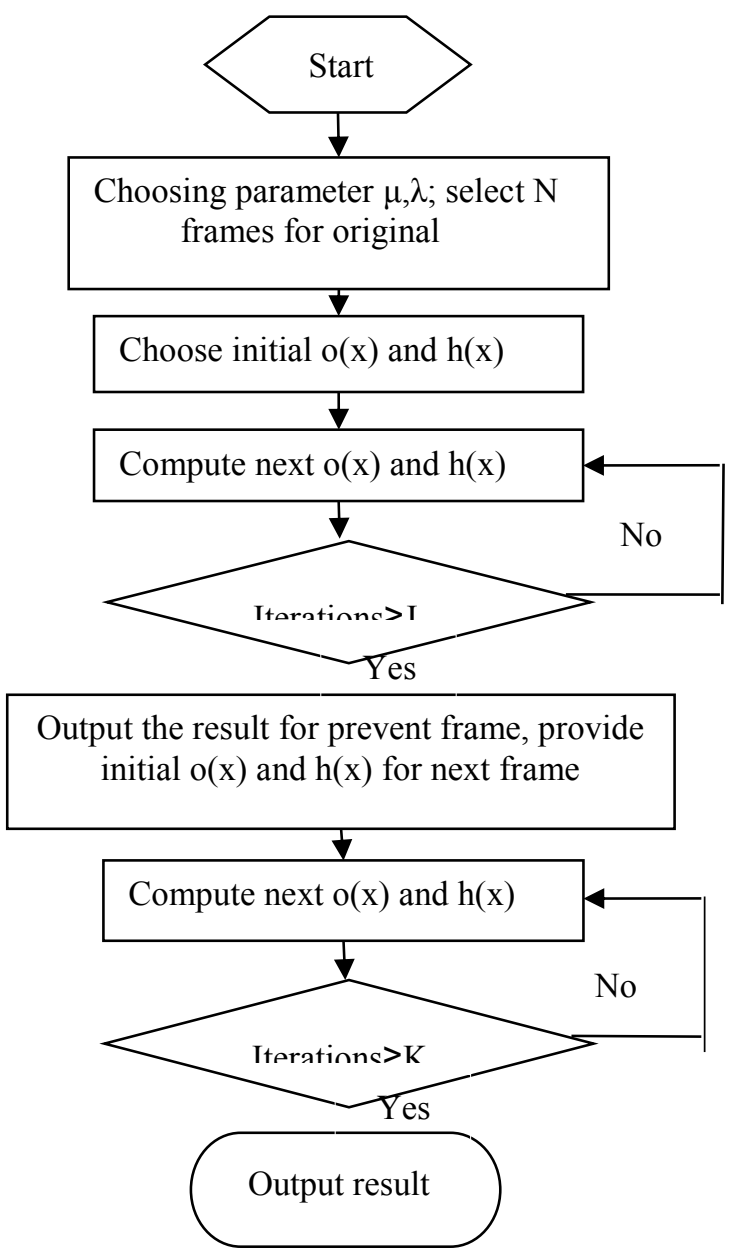

Fig.1. Flowchart of the proposed iterative algorithm

\section{RESULTS}

Fig.5 (a), (c), (e) are the frames of the video sequence, which are sheared to $256 * 256$ pixels. Fig. 5 (a) is the first frame of the sequence, and the previous five frames were selected as the original frame sequence in the experiment.

Let $\mu=0.25, \lambda_{1}=2 * 10^{3}, \lambda_{2}=4 * 10^{4}$, and the formula (5) and (6) was used to iterate 100 times, then we obtain the result. Fig.2 (b) is the restoration of the Fig.2 (a), this work in the first step of the two-step iterative algorithm. The deblurred image enhanced little compared to the blurred image, the fewer iterative times and worse initial estimate cause the result. Fig.2 (c) is the 12 frame of the sequence, the image work in the second step of the two-step iterative algorithm. We only use 20 iterations to compute the result. Fig.2 (d) is degraded from the Fig.2 (c), we may find that compared to the Fig.2 (c), the clarity of Fig.2 (d) obviously be promoted. Fig.2 (e) is the 35 frame of the sequence, and Fig.2 (f) is the restoration of the Fig.2 (e). The recovery is fine and effective, before recovery, we can't see clearly the number 126 in the plane in the Fig.2 (e). However, after the recovery, the font and the sign in the plane are clearly visible. Although the effect of the previous frames is not very good, the estimate of the target image is more accurate with the recovery of depth.

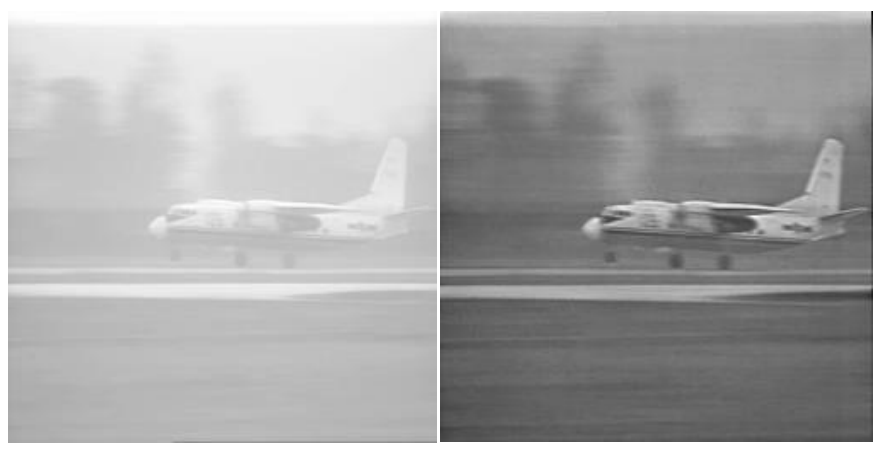

(a)

(b)

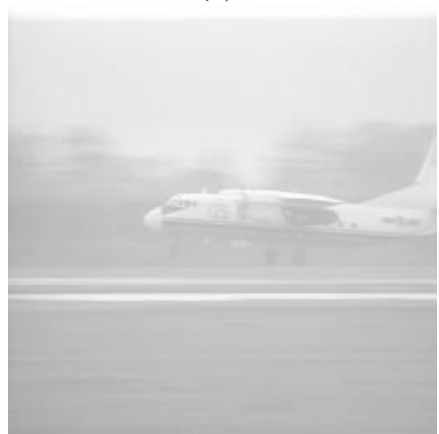

(c)

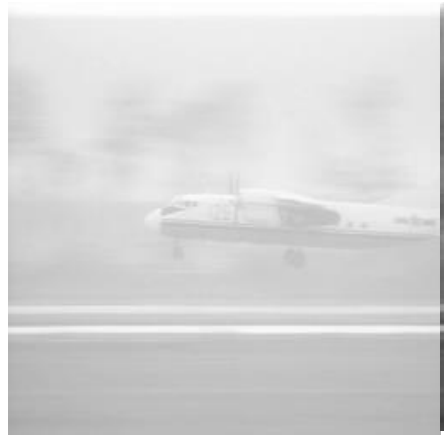

(e)

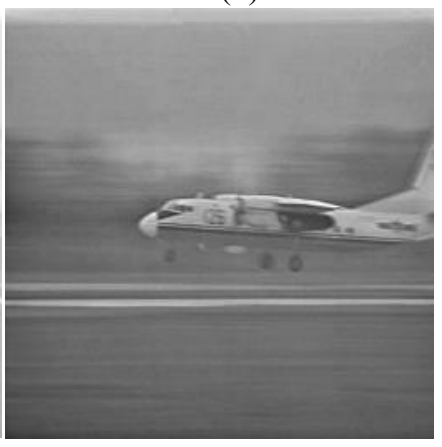

(d)

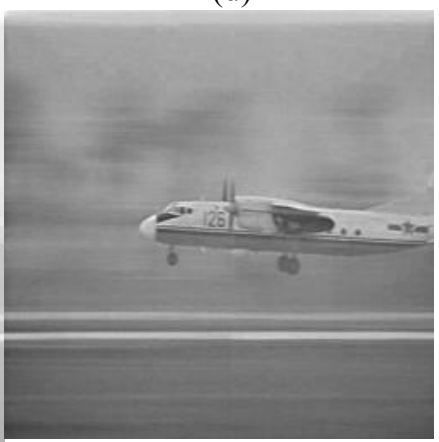

(f)
Fig.2. The image (a),(c),(e) are taken from the 1,12,35 frame of the video separately, the image (b),(d),(f) is deblured from the image(a),(c),(e).

In the algorithm, the computation time is mostly concentrated on the FFT operation, there are at least six FFT operations at each iteration, and the computation in each iteration are all the same. Above this we can consider the complexity of the algorithm is $\mathrm{O}(\mathrm{n} \log )$. For the image of $256 * 256$, we can get the computation time of the each iteration, about 0.04 second. The time of deblurring is proportionate to the iterative times. The most advantage of the two-step algorithm is that it can take 
little iterative times to obtain better image. The Fig. 3 can explain this point. Fig. 3 is the curve of iteration, the horizontal axis represents the iterative times, and the $\mathrm{Y}$-axis represents the residual. The black line represents the direct iterative algorithm, and the green line represents the proposed iterative algorithm. The black line with red sign is the restoration of the first frame by the iterative algorithm.

From the picture, the curve of first frame convergent slowly, after 50 times of iteration, the curve still don't convergence. Even more, the residual is very large. This is the reason that the effect of the first frame promoted not much, so in the first step of the algorithm, we need adopt more iterations. Compared to the black line, the green line not only has smaller residual, but also convergent quickly, only 20 times of iteration, it is converged to a less residual. From the results show that the proposed algorithm can convergence quickly and get a better result.

Fig.4 shows the normalized gray value. By selecting four independent pixels in the image, we can find that every curve approaches a constant after a certain iteration. Four pixels in different position were selected, each picture is the gray value after the normalized in every iteration. The horizontal direction means the iterative times, the vertical direction means the gray value.
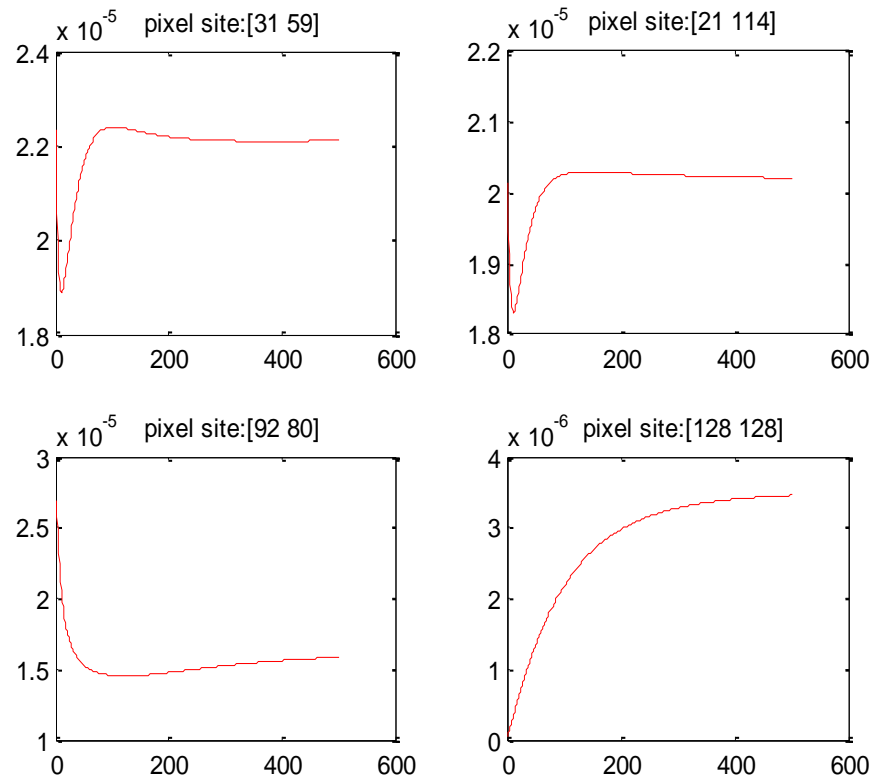

Fig.4. The gray value after the normalized in every iteration

Though ultimate value is different from every pixel, we can see these iterative values consist with the optimal estimation of the deblurred image. From this point, we can find another advantage of the algorithm.

Fig. 5 is the contrast between the algorithm in the article and the other universal algorithm, Fig5.a is the blurred image. Fig5.b is the image deblurred by the method in this essay. Fig5.c is the image deblurred by the MIA algorithm. Fig5.d is the image deblurred by Wiener-IBD.

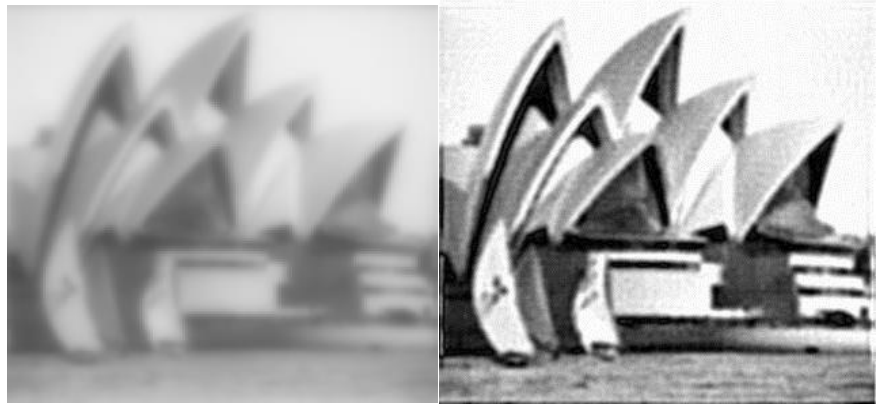

(a)

(b)

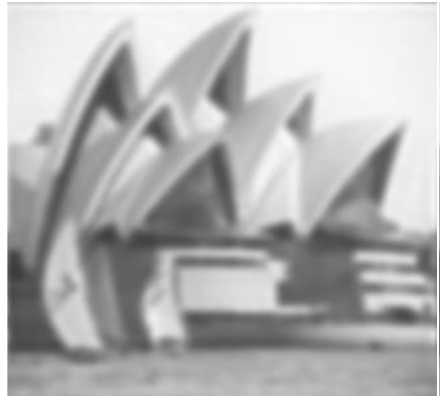

(c)

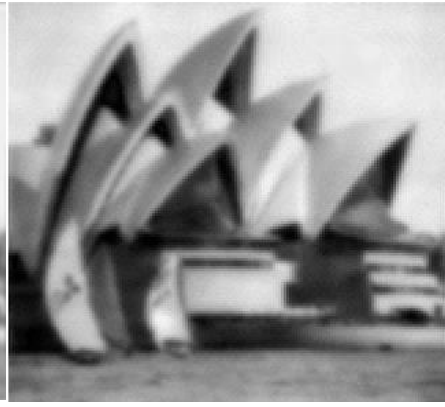

(d)

Fig.5. The contrast algorithms

Fig6 is the contrast between the algorithm in the article and the other based on MAP estimate and iterative algorithm. According to the source image [19], we give the evaluate value about SSD and err ratio. The result shows, for the single image, our iterative method also have fine performance.

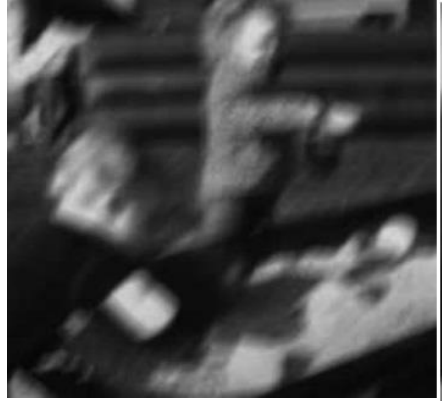

(a) the input degraded image

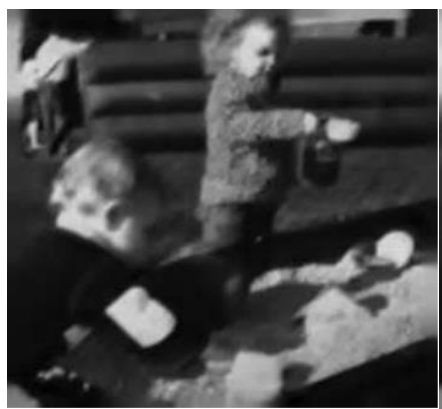

(c) $\mathrm{SSD}=53.6$,err ratio $=1.44$

\section{CONCLUSION}

Towards the short-exposure or high rate of frame image, there is a feature that the target image is invariant in a shortterm. Bring in this prior to the image deblurring, we adopt the 
result of the previous frame as the starting estimate in current frame. In this way, we can shorten the iterative path and get convergence quickly. The result shows the algorithm own the faster convergence speed and the better convergence result. Different from the other algorithm for blind restoration, although we should select the parameter in the starting of the algorithm, the algorithm doesn't sensitive for the parameter. So the algorithm possesses very strong adaptability for the blind image deblurring. Furthermore, benefited from the interframe information, the neighbouring frame can be seen as degenerated from the same object image and different point spread function (PSF), so utilizing the result of the last frame to the initial estimate of the current frame can reduce iterative times and enhance the efficiency of the algorithm.

As for the choice of distribution fitting model, it is not enough to improve the speed of image deconvolution only by using fast Fourier transform. For the convenience of research and implementation, Gaussian distribution can be used to fit the natural image gradient distribution, but the super Laplacian distribution model is the most complex, but the effect of fitting with the natural image gradient distribution is the best, so the super Laplacian distribution model can be used to fit the natural image gradient distribution in the future research direction. Furthermore, we can propose more complex distribution models, such as Gaussian distribution and super Laplacian distribution to fit natural images. With the increase of computation, we can choose the algorithm with faster iteration speed to achieve.

\section{ACKNOWLEDGEMENTS}

This work is supported by the Academician Workstation of Taiyuan Institute of Technology (TYSYSGZZ201903).

\section{REFERENCES}

[1] Latinovic N, Vukovic T, Petrovic R, et al. "Implementation Challenge and Analysis of Thermal Image Degradation on R-CNN Face Detection", Telfor Journal, 2020, 12, pp. 98-103.

[2] Khanam Z, Aslam B, Saha S, et al. "Gamma-Induced Image Degradation Analysis of Robot Vision Sensor for Autonomous Inspection of Nuclear Sites”. IEEE Sensors Journal, 2021, PP(99):1-1.

[3] Mikel A , Alejandro M A , JFB Ramírez, et al. "Tear film stability assessment by corneal reflex image degradation: publisher's note. Journal of the Optical Society of America, 2020, 37, pp.888.

[4] Chalvidal M, Chouzenoux E. "Block Distributed Majorize-Minimize Memory Gradient Algorithm and its application to 3D image restoration", 2020, 73, pp. 254-267.

[5] He N A, Wang R B, Wang Y C . "Dynamic MRI reconstruction exploiting blind compressed sensing combined transform learning regularization ScienceDirect", Neurocomputing, 2020, 392, pp. 160-167.

[6] Peper E S, Gottwald L M, Zhang Q, et al. "Highly accelerated 4D flow cardiovascular magnetic resonance using a pseudo-spiral Cartesian acquisition and compressed sensing reconstruction for carotid flow and wall shear stress", Journal of Cardiovascular Magnetic Resonance, 2020, 22, pp. 2278-2324.

[7] Kavitha T S , Prasad K S . "Hybridizing ant lion with whale optimization algorithm for compressed sensing MR image reconstruction via 11 minimization: an ALWOA strategy", Evolutionary Intelligence, 2020, 7, pp.1-11.

[8] Gharamohammadi A, Behnia F, Shokouhmand A, et al. "Robust Wiener filter- based time gating method for detection of shallowly buried objects", IET Signal Processing, 2021, 15, pp.665-678.
[9] Deekshit K, Madhav G V . "Performance Analysis Of Wiener Filter With Different Window Functions In Detecting Broken Rotor Fault In 3 Phase Induction Motor". International Journal of Engineering Trends and Technology, 2020, 68, pp.153-159.

[10] Jeong Y J , Park H S , Ji E J , et al. "Restoration of amyloid PET images obtained with short-time data using a generative adversarial networks framework", Scientific Reports, 2021, 11, pp.4825.

[11] Kumar S , Kosa J A , Luhach A K , et al. "Editorial: Advances in Computational Intelligence for Machine Vision and Image Processing", International Journal of Intelligent Engineering Informatics, 2020, 8, pp.263-267.

[12] Adly A A , Abd-El-Hafiz S K . "Utilizing four-node tetrahedra-shaped Hopfield neural network configurations in the local magnetization assessment of 3d objects exhibiting hysteresis", AIP Advances, 2021, 11, pp.025018.

[13] Yang F, Wang X. "Dynamic characteristic of a new fractional-order chaotic system based on the Hopfield Neural Network and its digital circuit implementation" Physica Scripta, 2021, 96, pp. 035218.

[14] Cacciari I , Mugnai D , Ranfagni A, et al. "Cross-modulation between microwave beams interpreted as a stochastic process", International Journal of Modern Physics B, 2021, 121, pp.1156.

[15] Onodera M , K Aratani, Shonai T, et al. "Lateral Position With Gantry Tilt Further Improves Computed Tomography Image Quality Reconstructed Using Single-Energy Metal Artifact Reduction Algorithm in the Oral Cavity", Journal of Computer Assisted Tomography, 2020, 44, pp. 553-558

[16] Murase K. "New image-restoration method using a simultaneous algebraic reconstruction technique: comparison with the Richardson-Lucy algorithm", Radiological Physics and Technology, 2020, 13, pp. 365-377.

[17] Y Qi, P Zhang, Y Chen, "Probability of Signal Demodulation Jump Errors inMaximum-likelihood-estimation algorithm for lowSNR interference spectrum", Applied Optics, 2021, 60, pp. 93-102.

[18] Alcaraz J , Anton-Sanchez L , Aparicio J , et al. "Russell Graph Efficiency Measures in Data Envelopment Analysis: the Multiplicative Approach", European Journal of Operational Research, 2020, 34, pp. 238-249.

[19] Yamaguchi M , Yamamoto S , Kitano M, et al. "Estimation of shifts of therapeutic carbon-ion beams owing to cavities in a polyethylene target by measuring prompt X-ray images", Japanese Journal of Applied Physics, 2020, 59, pp. 77-90.

[20] Dhont, Verellen D , Mollaert I, et al. "RealDRR - Rendering of realistic digitally reconstructed radiographs using locally trained image-to-image translation", Radiotherapy and Oncology, 2020, 153, pp. 352-360.

[21] Rafeek Mamdouh, Hazem M. El-Bakry, Alaa Riad, Nashaat El-Khamisy, Converting 2D-Medical Image Files into 3D- Models, based on Image Processing, and Analysing their Results with Python Programming, WSEAS Transactions on Computers, Volume 19, 2020, Art. \#2, pp. 10-20.

[22] Sergey, Stankevich, Mikhail, Popov, Sergiy V. Shklyar, Konstantin Y. Sukhanov, Artem, Andreiev, Artur R. Lysenko, Xing Kun, Cao Shixiang, Shi Yupa, Zhang Xing, Sun Boya, Subpixel-shifted Satellite Images Superresolution: Software Implementation, WSEAS Transactions on Computers, Volume 19, 2020, Art. \#5, pp. 31-37.

\section{Creative Commons Attribution License 4.0 (Attribution 4.0 International, CC BY 4.0)}

This article is published under the terms of the Creative Commons Attribution License 4.0

https://creativecommons.org/licenses/by/4.0/deed.en_US 


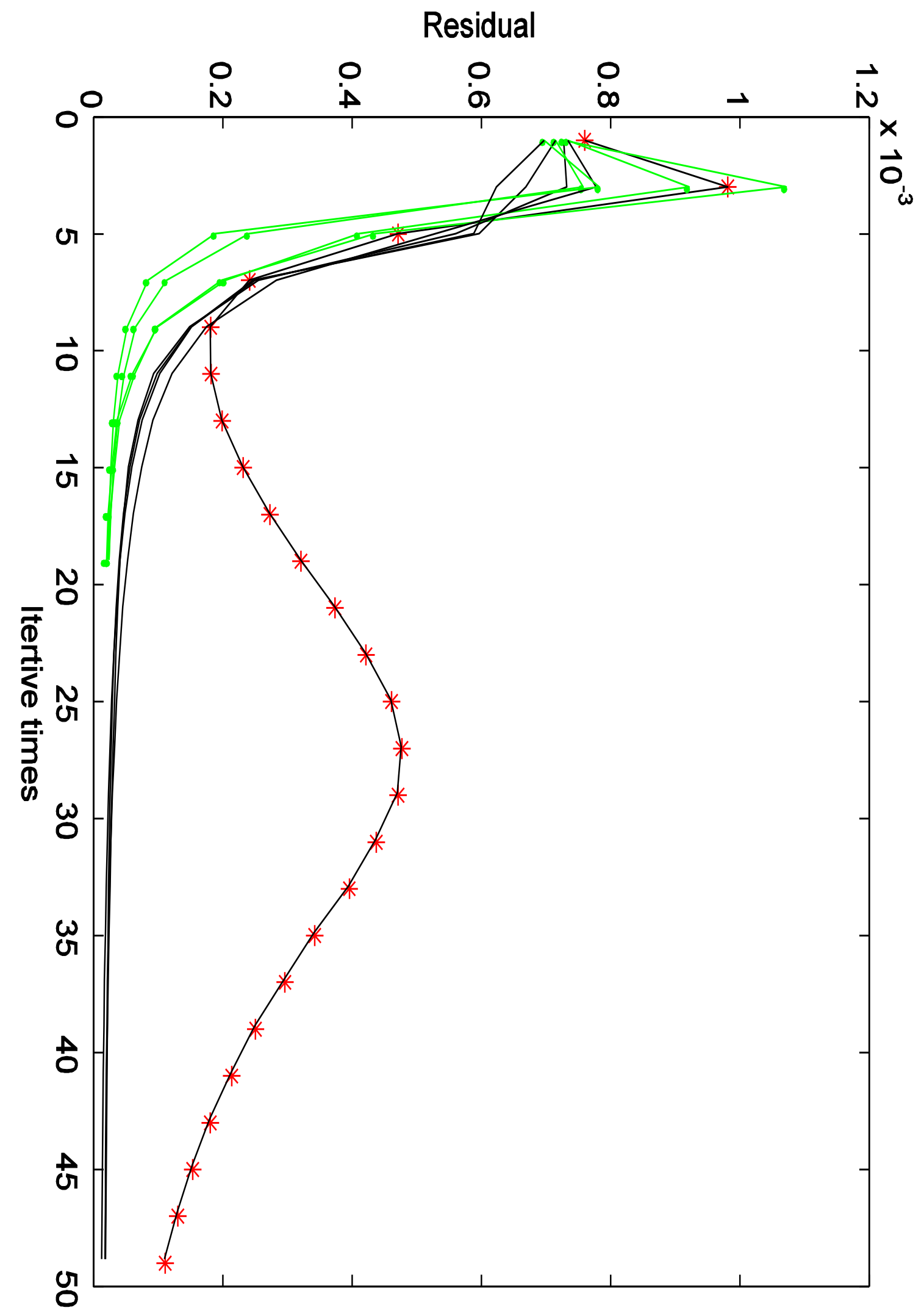

Fig.3. The curve of convergence by iterations 